\title{
Magnesium Sheet Technology Perspectives
}

\author{
Elhachmi Essadiqi
}

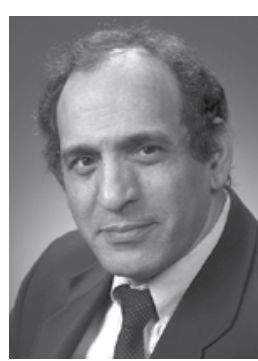

The automotive industry is facing a significant challenge in reducing vehicle weight to improve fuel economy and reduce greenhouse gas emissions without compromising safety. Vehicle weight reduction is best accomplished through a combination of innovative structural design and increased use of lightweight materials. Magnesium alloys, which have high specific strength, are very attractive materials for weight reduction in automotive applications. At present, magnesium use in the auto industry is limited to die-cast parts, although wrought magnesium alloys hold great promise for use in structural applications. However, the use of AZ31 magnesium sheet is limited by low ductility at room temperature, high production cost, and high corrosion rates. To compete with other structural materials, such as steel and aluminum, magnesium must overcome these obstacles. In this context, extensive research and development on magnesium sheet is underway worldwide. It includes twinroll casting, hot rolling, forming, welding, and crashworthiness testing. It was found that the magnesium sheet produced by twin-roll casting is more economical than that presently produced using direct chill casting. The twin-roll casting process is a mature technology for aluminum production, but this is not so for magnesium, where development work is needed. Several research centers around the world-in Korea, China, Germany, Australia, Japan, and Canada - are focusing on the development of twin-roll casting of magnesium.
Another challenge is the development of a new magnesium alloy that is formable at room temperature or at least at temperatures lower than $250^{\circ} \mathrm{C}$. Extensive research is being carried out worldwide in alloy design, examining different magnesium alloy systems with and without aluminum and including additions of rare earth (RE) and other alloying elements. Thermodynamic calculations and phase diagrams of various magnesium alloy systems are essential to alloy development. Extensive work is being done in this area in Canada and Germany. The texture of hot-rolled magnesium sheet is of great importance to its formability characteristics. The focus of this research is on production of magnesium sheet with random texture avoiding basal texture components that reduce magnesium formability.

The fabrication of auto parts from magnesium alloys requires development of enabling technologies, such as joining, forming, and coating. Magnesium sheet alloys have to be joined to other automotive parts made of either similar material or dissimilar materials such as steel and aluminum. Forming and crashworthiness are other technologies to be developed for magnesium, and coating development is also required for protection against corrosion.

In this issue, Park et al. review recent progress in the twin-roll casting of magnesium alloys, focusing on the processing aspects that are closely related to the solidification behavior of magnesium alloy strips. Recent attempts to develop new magnesium alloys utilizing the metallurgical advantages attainable by this novel casting process are covered.

Barnett et al. outline some of the barriers to achieving improved formability of magnesium alloys at ambient temperature. The underlying metallurgical characteristics of the issues are considered, and means for overcoming them are discussed. It is concluded that a combination of microstructure and texture control is a promising strategy.

Essadiqi et al. present the effect of rolling schedule on microstructure of AZ31 magnesium sheet, the evolution of microstructure during hot rolling by interrupted-rolling experiments, and finally, modeling of hot rolling based on a thermal-mechanical coupling technique in a finite element program for hot rolling schedule design and optimization.

Verma et al. describe the application of a new material constitutive model in finite element simulations for the hot forming of magnesium AZ31 alloy sheet. Simulations of forming both simple geometries in laboratory studies and complex parts in production trials are presented and compared with experimental results.

Hantzsche et al. address the relationship between microstructure, texture, and mechanical properties of rolled magnesium sheets. The effects of rolling temperature and alloying elements on texture development and mechanical properties are demonstrated. Particular focus is placed on the potential of rare earth elements to modify the anisotropic behavior and to weaken the strong basal texture of magnesium sheets. A combination of alloy design and process characteristics is required to produce magnesium sheets with improved forming properties.

Elhachmi Essadiqi is with the Materials Technology Laboratory-CANMET in Ottawa, Ontario, Canada and is the advisor to JOM from the Magnesium Committee of the Light Metals Division of TMS. 